\title{
Identification of Bioactive Components from Ruellia tuberosa L. on Improving Glucose Uptake in TNF- $\alpha$-Induced Insulin-Resistant Mouse FL83B Hepatocytes
}

\author{
Jian-Hua Xu, ${ }^{1}$ Yangming Martin Lo, ${ }^{2}$ Wen-Chang Chang, ${ }^{3}$ Da-Wei Huang, ${ }^{4}$ \\ James Swi-Bea Wu, ${ }^{5}$ Yu-Yuan Jhang, ${ }^{6}$ Wen-Chung Huang, ${ }^{7}$ Chih-Yuan Ko $\mathbb{D}^{8,}{ }^{8,9,10,11}$ \\ and Szu-Chuan Shen (iD ${ }^{6}$ \\ ${ }^{1}$ Department of Tumor Surgery, The Second Affiliated Hospital of Fujian Medical University, Quanzhou 362000, China \\ ${ }^{2}$ Institute for Advanced Study, Shenzhen University, Shenzhen 518060, China \\ ${ }^{3}$ Department of Food Science, National Chiayi University, Chiayi City 60004, Taiwan \\ ${ }^{4}$ Department of Biotechnology and Food Technology, Southern Taiwan University of Science and Technology, \\ Tainan City 71005, Taiwan \\ ${ }^{5}$ Graduate Institute of Food Science and Technology, National Taiwan University, Taipei 10672, Taiwan \\ ${ }^{6}$ Graduate Program of Nutrition Science, National Taiwan Normal University, Taipei 10610, Taiwan \\ ${ }^{7}$ Graduate Institute of Health Industry Technology, Chang Gung University of Science and Technology, Taoyuan 33303, Taiwan \\ ${ }^{8}$ Department of Respiratory and Critical Care Medicine, The Second Affiliated Hospital of Fujian Medical University, \\ Quanzhou 362000, China \\ ${ }^{9}$ Department of Clinical Nutrition, The Second Affiliated Hospital of Fujian Medical University, Quanzhou 362000, China \\ ${ }^{10}$ School of Public Health, Fujian Medical University, Fuzhou 350122, Fujian, China \\ ${ }^{11}$ Respiratory Medicine Center of Fujian Province, Quanzhou 362000, China
}

Correspondence should be addressed to Chih-Yuan Ko; yuanmomoko@gmail.com and Szu-Chuan Shen; scs@ntnu.edu.tw

Received 21 October 2020; Revised 11 November 2020; Accepted 18 November 2020; Published 1 December 2020

Academic Editor: Armando Zarrelli

Copyright (C) 2020 Jian-Hua Xu et al. This is an open access article distributed under the Creative Commons Attribution License, which permits unrestricted use, distribution, and reproduction in any medium, provided the original work is properly cited.

Ruellia tuberosa L. (RTL) has been used as a folk medicine to cure diabetes in Asia. RTL was previously reported to alleviate hyperglycemia, insulin resistance (IR), abnormal hepatic detoxification, and liver steatosis. However, the potential bioactive compounds of RTL have still not been identified. The aim of this study was to investigate the bioactive compounds in RTL ethyl acetate (EA) fractions by using a glucose uptake assay in TNF- $\alpha$-treated mouse FL83B hepatocytes to discover a mechanism by which to improve IR. The bioactive compounds were identified by the high-performance liquid chromatography (HPLC) assay. Using the Sephadex LH20 gel packing chromatography column, the EAF5 fraction was isolated from RTL and significantly increased glucose uptake in TNF- $\alpha$-treated FL83B cells. Moreover, the MCI gel packing chromatography column separated EAF5 into five subfractions and had no significant cytotoxic effect in FL83B cells when treated at the concentration of $25 \mu \mathrm{g} / \mathrm{ml}$. Among the subfractions, EAF5-5 markedly enhanced glucose uptake in TNF- $\alpha$-treated FL83B cells. The possible bioactive compounds of the EAF5-5 fraction that were identified by the HPLC assay include syringic acid, $p$-coumaric acid, and cirsimaritin. The bioactive compound with the best effect of increasing glucose uptake was $p$-coumaric acid, but its effect alone was not as good as the combined effect of all three compounds of the EAF5-5 fraction. Thus, we speculate that the antidiabetic effect of RTL may be the result of multiple active ingredients. 


\section{Introduction}

Type 2 diabetes mellitus (T2DM) is characterized by insulin resistance (IR), which is caused by insufficient production of insulin or by the ineffectiveness of insulin activity to maintain constant blood sugar. In response to hyperglycemia, the presence of high levels of blood sugar, the body compensatively secretes more blood insulin in increasing concentrations, causing hyperinsulinemia. Once the body presents hyperglycemia and hyperinsulinemia, it can be prone to many complications [1]. Diabetic control and therapy can consist of three parts, including dietary therapy, exercise, and medications. Unfortunately, most T2DM patients will depend on drug treatments, which have adverse side effects. Diabetic drugs have been reported to cause nausea, abdominal pain, or weight gain [2, 3]. Recently, researchers have turned to natural plant ingredients containing phenolic acids or flavonoids. Moreover, various ingredients have been proven to alleviate hyperglycemia or antidiabetic effects [4-6].

Ruellia tuberosa L. (RTL) is a herb plant that has been used as folk medicine to cure diabetes in Asian countries for decades. Our previous studies have demonstrated that RTL aqueous or ethanolic extracts can significantly improve glucose uptake in $\mathrm{C} 2 \mathrm{C} 12$ myoblasts, alleviate the tumor necrosis factor (TNF)- $\alpha$-induced IR in skeletal muscles, and ameliorate hyperglycemia, IR indices, and aorta dysfunction in high-fat-diet-fed streptozotocin-induced T2DM rats [7, 8]. Additionally, RTL ameliorated abnormal hepatic detoxification, nonalcoholic fatty liver disease, and lipid accumulation in the liver of T2DM rats $[9,10]$.

Previous studies have identified more than sixty compounds in RTL. The compounds obtained by separation and purification are mainly flavonoids, triterpenes, and steroids also containing long-chain fatty acids, alkaloids, and lignans [11-13]. However, there remains a void in the literature regarding the role of these potent RTL compounds alleviating hepatic glucose uptake. Therefore, we aimed to screen the best glucose uptake activity of TNF- $\alpha$-induced IR in FL83B hepatocytes with ethyl acetate (EA) fractions of RTL by using thin-layer chromatography (TLC) and high-performance liquid chromatography (HPLC) to identify and characterize the specific potent compounds.

\section{Materials and Methods}

2.1. Preparation of RTL Extracts. The extraction protocol was based on our previous methods, with slight modifications $[7,9]$. One gram of RTL stems and leaves were extracted with $6 \mathrm{ml}$ distilled water and methanol $(1: 6, w / v)$ at $4^{\circ} \mathrm{C}$ for $72 \mathrm{~h}$ and filtered using a cheese cloth. The filtrate was filtered twice through a Whatman No. 1 filter paper and centrifuged at $7,000 \times \mathrm{g}$ for $20 \mathrm{~min}$. The supernatant was vacuum concentrated using a rotary evaporator below $40^{\circ} \mathrm{C}$. The concentrated methanol extract was dissolved in $400 \mathrm{ml}$ water and subsequently extracted with an equal volume of hexane and EA solvent, and the EA layers were divided through column chromatography using a Sephadex LH-20 column with $200 \mathrm{ml}$ of $50-100 \%$ methanol sequentially. The different fractions were then collected from the EA layer (EAF1 to EAF8). The best of glucose uptake hepatocytes of fractions was evaluated and screened, and the EAF5 fractions were divided through column chromatography using an MCI gel column with $200 \mathrm{ml}$ of $20-100 \%$ methanol sequentially. The different fractions were then collected from the EAF5 layer (EAF5-1 to EAF5-5), as shown in Figure 1.

2.2. TLC. The supernatants were placed onto a silica gel precoated plate (Kieselgel 60 F254, $0.20 \mathrm{~mm}$, Merck, Darmstadt, Germany). The TLC plates were added with a solvent mixture of dichloromethane:methanol:water: acetic acid $(10: 1: 0.1: 0.2, v / v)$, followed by immersion into $10 \%$ sulfuric acid, and then the mixture was heated. Using the color distribution state of TLC, similar effluents were gathered, and the solvent was drained through a rotary evaporator. Different concentrates were freeze-dried into a powder and kept at $-80^{\circ} \mathrm{C}$ until use.

2.3. Cell Culture. The experiments were performed on FL83B mouse hepatocytes (ATCC, Rockville, MD, USA) incubated in $\mathrm{F} 12 \mathrm{~K}$ medium containing $10 \%$ fetal bovine serum (Invitrogen Corporation, Camarillo, CA, USA) in $10 \mathrm{~cm}$ Petri dishes at $37^{\circ} \mathrm{C}$ and $5 \% \mathrm{CO}_{2}$. The experiments were conducted on cells that were $80-90 \%$ confluent.

2.4. TNF- $\alpha$ Induction of IR in FL83B Cells. IR was induced according to a method described previously [7, 14]. FL83B cells were seeded in $10 \mathrm{~cm}$ dishes and incubated at $37^{\circ} \mathrm{C}$ for $48 \mathrm{~h}$ to reach $80 \%$ confluences. Serum-free medium containing recombinant mouse TNF- $\alpha$ and different RTL fractions $(25 \mu \mathrm{g} / \mathrm{ml})$ was incubated for $16 \mathrm{~h}$ at $37^{\circ} \mathrm{C}$ to induce IR.

2.5. Uptake of Fluorescent 2-[N-(7-Nitrobenz-2-oxa-1,3-diazol4-yl) amino]-2-deoxy-d-glucose (2-NBDG) in FL83B cells. The FL83B cells were seeded in $10 \mathrm{~cm}$ dishes and then incubated at $37^{\circ} \mathrm{C}$ for $48 \mathrm{~h}$ to achieve $80 \%$ confluences. Serumfree medium containing $20 \mathrm{ng} / \mathrm{ml}$ recombinant mouse TNF$\alpha$ was added before incubating for $16 \mathrm{~h}$ to induce IR. The cells were then transferred to another F12K medium containing $5 \mathrm{~mm}$ glucose, without (basal) or with $200 \mu \mathrm{m}$ insulin and $10 \mu \mathrm{l}$ different RTL fractions and incubated for $30 \mathrm{~min}$ at $37^{\circ} \mathrm{C}$. An assay of glucose uptake was then performed as described previously [7]. The fluorescence intensity of the cell suspension was evaluated using flow cytometry (FACScan, Becton Dickinson, Bellport, NY, USA) at an excitation wavelength of $488 \mathrm{~nm}$ and an emission wavelength of $542 \mathrm{~nm}$. Fluorescence intensity reflected the cellular uptake of 2-NBDG.

2.6. Chemical Profiles. The total phenolic and flavonoid contents of EA extracts were determined by the HPLC assay $[15,16]$. Gallic acid, protocatechuic acid, vanillic acid, syringic acid, caffeic acid, sinapic acid, ferulic acid, $p$-coumaric acid, and cinnamic acid were chosen as standards for 


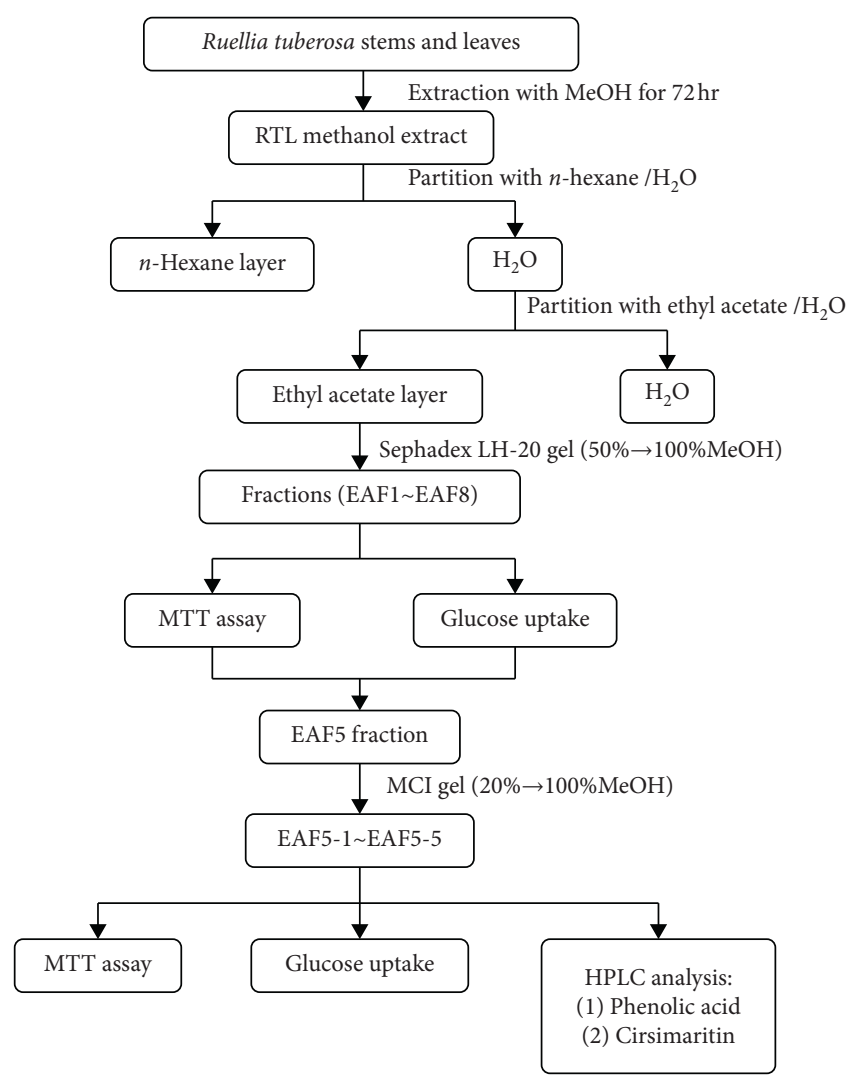

FIGURE 1: The flowchart for fractionation of Ruellia tuberosa Linn. (RTL) extraction.

phenols, whereas cirsimaritin was chosen as the standard for flavonoids. The samples $(10 \mathrm{mg})$ were dissolved in $1 \mathrm{ml}$ methanol and filtered through a $0.22 \mu \mathrm{m}$ filter. The HPLC system was equipped with a diode array detector (Shimadzu, Kyoto, Japan) and Varian Polaris C18 column $(5 \mu \mathrm{m}$, $250 \mathrm{~mm} \times 4.6 \mathrm{~mm}$; Agilent, CA, USA). The injection volume was $10 \mu \mathrm{l}$ with a flow rate of $1.0 \mathrm{ml} / \mathrm{min}$. For the phenolic acid assay, the mobile phases were methanol (eluent $\mathrm{A}$ ) and acetic acid (eluent $B$ ) in the following gradient elutions: $5 \%$ (A), $95 \%$ (B) in $0-5 \mathrm{~min}$; $5 \%-20 \%(\mathrm{~A}), 95 \%-90 \%$ (B) in 5-10 min; $20 \%-40 \%$ (A), $90 \%-60 \%$ (B) in $10-20$ min; $40 \%-$ $60 \%(\mathrm{~A}), 60 \%-40 \%(\mathrm{~B})$ in $20-30 \mathrm{~min}$; and $60 \%-100 \%(\mathrm{~A})$, $40 \%-0 \%$ (B) in $30-40 \mathrm{~min}$. The samples were detected by a UV detector $(280 \mathrm{~nm})$. For the flavonoid assay, the mobile phases were methanol (eluent A) and acetic acid (eluent B) in the following gradient elutions: $50 \%-100 \%(A), 50 \%-0 \%$ (B) in $0-25 \mathrm{~min}$. The samples were detected by using a UV detector $(270 \mathrm{~nm})$. The standards for phenolic acids and cirsimaritin (purchased from Sigma, St Louis, MO, USA) had a coefficient of determination $\left(r^{2}\right)$ that was greater than 0.995 .

2.7. Statistical Analyses. Values are presented as the mean \pm standard deviation using SPSS version 22.0 (SPSS Inc., Chicago, IL, USA) by one-way analysis of variance (ANOVA) and Duncan's new multiple range tests. $P<0.05$ was considered statistically significant.

\section{Results}

3.1. Effect of Different EA Fractions from RTL on Glucose Uptake in FL83B Mouse Hepatocytes. The different RTL fractions from the EA layers (EAF1 to EAF8) were collected through TLC analysis (Supplementary Figure S1). When the concentration of each fraction was less than $25 \mu \mathrm{g} / \mathrm{ml}$, the cell survival rate was greater than $80 \%$ and did not inhibit cell growth (Supplementary Figure S2). Thus, the concentration of $25 \mu \mathrm{g} / \mathrm{ml}$ was used for the follow-up glucose uptake evaluation.

An evaluation of the 2-NBDG uptake was performed to assess the improvement of glucose uptake in FL83B hepatocytes. Compared with the TNF- $\alpha$-treated group, the fluorescence content of the two fractions of EAF5 and EAF7 was significantly increased, and the fluorescence intensity was $93.3 \pm 5.4 \%$ and $88.9 \pm 2.8 \%(P<0.05)$, respectively. The EAF5-treated group demonstrated the best improvement in glucose uptake in the IR-induced FL83B hepatocytes. Thus, EAF5 was used for the following examinations (Figure 2).

3.2. Effect of Different EAF5 Fractions from RTL on Glucose Uptake in FL83B Mouse Hepatocytes. The different EAF5 fractions (EAF5-1-EAF5-5) were collected through TLC analysis (Supplementary Figure S3). MTT assay showed that the optimal concentration was $25 \mu \mathrm{g} / \mathrm{ml}$ for cell survival of EAF5 subfractions (Supplementary Figure S4). The EAF5-5 fraction significantly increased fluorescence intensity of hepatocytes $(108.4 \pm 7.7 \%)$, compared with the TNF- $\alpha$-induced IR group $(P<0.05$; Figure 3$)$.

3.3. Bioactive Components of RTL. Nine standards of phenolic acid, including gallic acid, protocatechuic acid, vanillic acid, syringic acid, caffeic acid, sinapic acid, ferulic acid, $p$ coumaric acid, and cinnamic acid, and retention time (10.66, $17.15,23.06,23.66,24.09,27.70,28.24,28.78$, and $35.27 \mathrm{~min}$, respectively) are shown in Supplementary Figure S5. The standard of cirsimaritin was examined by HPLC, and the retention time was $16.91 \mathrm{~min}$, detail shown in Supplementary Figure S6. EAF5-5 contains $27.3 \pm 1.4 \mu \mathrm{g} / \mathrm{g}$ syringic acid, $95.0 \pm 2.5 \mu \mathrm{g} / \mathrm{g}$ p-coumaric acid, and $805.5 \pm 24.8 \mu \mathrm{g} / \mathrm{g}$ cirsimaritin (Table 1).

3.4. Evaluation of Bioactive Components from RTL on Glucose Uptake in IR of FL83B Hepatocytes. The fluorescence intensity of syringic acid, $p$-coumaric acid, and cirsimaritin was $97.4 \pm 20.8 \%, 98.8 \pm 13.7 \%$, and $87.1 \pm 4.3 \%$, respectively, and among them, $p$-coumaric acid has the most significant ability to improve glucose uptake, which can increase by about $22 \%$, compared with the TNF- $\alpha$ group $(P<0.05$; Figure 4).

The fluorescence intensity of the three bioactive components mixed (the mixing ratio was equal to the ratio contained in EAF5-5) and EAF5-5 was $120.9 \pm 5.1 \%$ and $118.1 \pm 16.5 \%$, which were significantly increased by $45 \%$ and $42 \%$, respectively, compared to the TNF- $\alpha$ group $(P<0.05$; Figure 4$)$. 


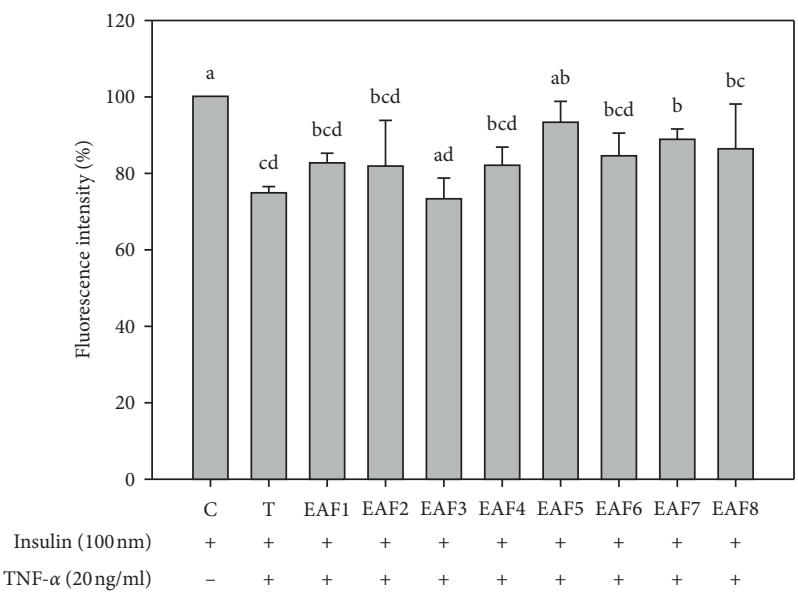

Figure 2: Effects of RTL-EA fractions on glucose uptake in mouse FL83B hepatocytes. Each value is mean \pm SD ( $n=3$ ). C (control): FL83B cells incubated with F-12K medium. T (TNF- $\alpha$ treatment): FL83B cells incubated with F-12K medium containing TNF- $\alpha$ (20 ng/ml) for 16 hours to induce insulin resistance. EAFs: TNF- $\alpha$ induced insulin resistance and then coincubated with RTL-EA fractions $(25 \mu \mathrm{g} / \mathrm{ml})$ for 30 min. ${ }^{*}$ Significantly different from control $(P<0.05)$.

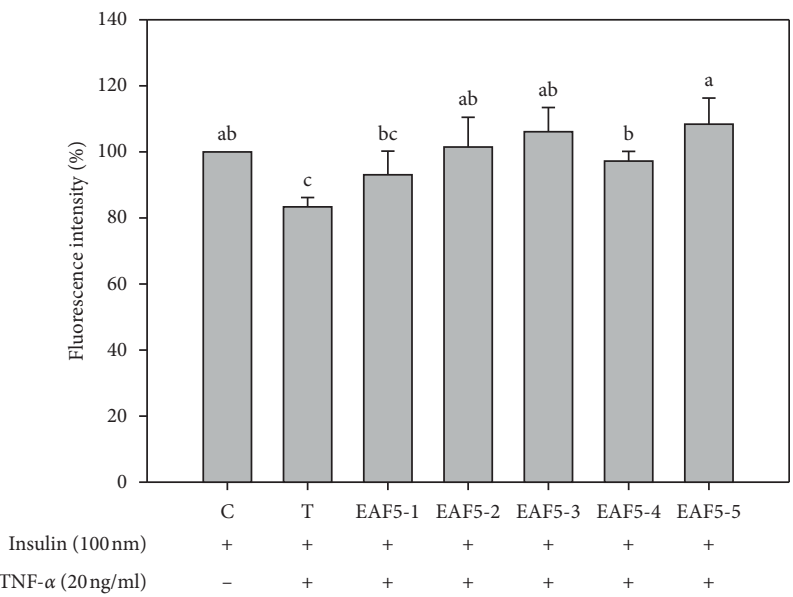

Figure 3: Effect of RTL-EAF5 fractions on glucose uptake in mouse FL83B hepatocytes. Each value is mean \pm SD ( $n=3)$. C (control): FL83B cells incubated with F-12K medium. T (TNF- $\alpha$ treatment): FL83B cells incubated with F-12K medium containing TNF- $\alpha$ (20 ng/ml) for 16 hours to induce insulin resistance. EAF5s: TNF- $\alpha$ induced insulin resistance and then coincubated with RTL-EAF5 fractions $(25 \mu \mathrm{g} / \mathrm{ml})$ for 30 min. * Significantly different from control $(P<0.05)$.

TABLE 1: Retention time and contents of phenolic acid and flavanoid from the RTL-EAF5 fraction.

\begin{tabular}{|c|c|c|c|c|c|c|}
\hline Standard & Retention time (min) & EAF5-1 $(\mu \mathrm{g} / \mathrm{g})$ & EAF5-2 $(\mu \mathrm{g} / \mathrm{g})$ & EAF5-3 $(\mu \mathrm{g} / \mathrm{g})$ & EAF5-4 $(\mu \mathrm{g} / \mathrm{g})$ & EAF5-5 $(\mu \mathrm{g} / \mathrm{g})$ \\
\hline \multicolumn{7}{|l|}{ Phenolic acid } \\
\hline Syringic acid & 23.66 & - & - & - & $6.7 \pm 0.9$ & $27.3 \pm 1.4$ \\
\hline$p$-Coumaric acid & 28.78 & - & - & - & - & $95.0 \pm 2.5$ \\
\hline \multicolumn{7}{|l|}{ Flavanoid } \\
\hline Cirsimaritin & 16.91 & - & - & - & - & $805.5 \pm 24.8$ \\
\hline
\end{tabular}

-, not detected. Each value is mean $\pm \mathrm{SD}(n=3)$.

\section{Discussion}

In this study, two columns, Sephadex LH-20 gel and MCI gel, were used to separate the bioactive components of the EA layer of RTL. The IR-induced FL83B hepatocytes model was used to screen out the distinguishing substances in the RTL that may improve glucose uptake, and then the active ingredients were identified by HPLC. Our results showed that in the first chromatography column of the EA layer of RTL, the EAF5 fraction significantly increased the glucose uptake of liver cells that induce IR by TNF- $\alpha$; thus, EAF5 was selected for the second chromatography column. EAF5-5 demonstrated the best potential activity for improving cellular glucose uptake based on the activity evaluation of the three types of bioactive phenolic acid and flavonoid compounds-syringic acid, $p$ coumaric acid, and cirsimaritin-which were identified by 


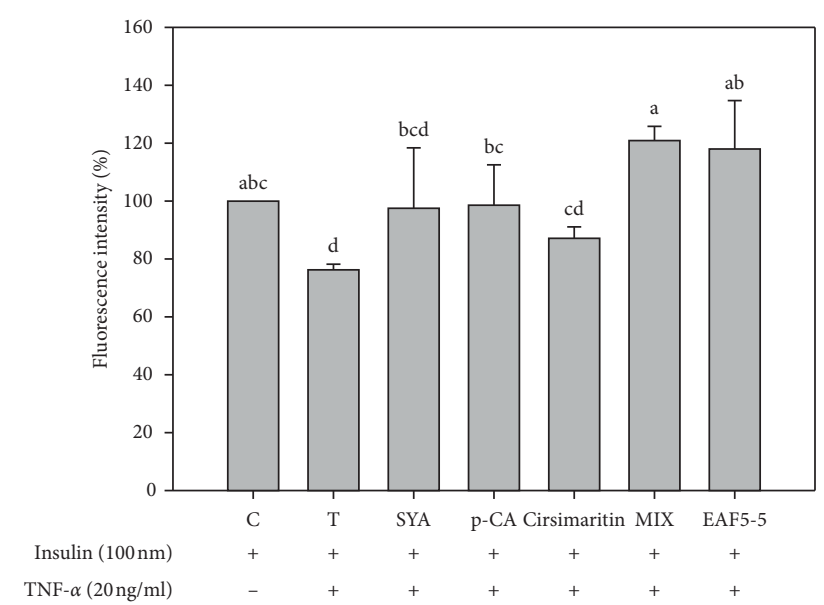

FIgURE 4: Effects of bioactive components in RTL on glucose uptake in mouse FL83B hepatocytes. Each value is mean \pm SD $(n=3)$. C (control): FL83B cells incubated with F-12K medium. T (TNF- $\alpha$ treatment): FL83B cells incubated with F-12K medium containing TNF- $\alpha(20 \mathrm{ng} / \mathrm{ml})$ for 16 hours to induce insulin resistance. SYA: syringic acid; p-CA: $p$-coumaric acid; MIX: syringic acid $+p$-coumaric acid + cirsimaritin. ${ }^{*}$ Significantly different from control $(P<0.05)$.

HPLC analysis. It has been suggested that phenolic compounds can significantly improve the activity of cellular glucose uptake, at least partially. Our results show that among the three active ingredients, $p$-coumaric acid is the most effective for increasing glucose uptake, but its effect is not as good as the combined activity of all three compounds and the EAF5-5 fraction.

An investigation of the cytotoxicity of the EA fractions in the cells was necessary before evaluating the cellular glucose uptake activity. In this study, when the concentration of all EA fractions from RTL was greater than $200 \mu \mathrm{g} / \mathrm{ml}$, it significantly inhibited cell growth in FL83B mouse hepatocytes. However, when the concentration was less than $25 \mu \mathrm{g} / \mathrm{ml}$ of each fraction, the cell survival rate was more than $80 \%$, which indicates that there was no significant inhibition of cell growth (Supplementary Figures S2 and S4). Hence, a concentration of $25 \mu \mathrm{g} /$ $\mathrm{ml}$ was used for each EAF fraction during the subsequent glucose uptake evaluation. Similarly, it was evident in our previous study that the concentration of RTL improved glucose uptake in mouse $\mathrm{C} 2 \mathrm{C} 12$ myoblasts [7].

Administration of RTL fractions of n-hexane and EA in diabetic rabbits lowered the animals' blood sugar levels [17]. Moreover, the best antioxidant activity result was reported from an evaluation of RTL extraction using methanol and EA [18]. RTL fractions of EA can effectively improve the blood sugar level in diabetes through their antioxidant capacity, as EA fractions speculatively contain high amounts of polyphenols and triterpenoids [17]. Another study indicated that the active compound of the EA fraction may be a polyphenol called cirsimaritin [19]. Our findings seem to be consistent with the above studies.

In this study, two phenolic acids, syringic acid and $p$ coumaric acid, were identified by HPLC, which had not been identified from RTL in the past. Previously, vanillic acid was isolated from an RTL extraction utilizing EA [20]. However, our present study did not identify vanillic acid from the fractions of RTL. It is speculated that when this compound cannot be identified, it is due to too little content or the extraction method that was used.

However, previous studies have confirmed that syringic acid and $p$-coumaric acid have many physiological activities, such as antioxidation, anticancer, and antibacterial [21-25]. Moreover, the effects of both of these compounds in treating diabetes have been demonstrated [26]. The administration of syringic acid in alloxan-induced diabetic rats for 30 days showed increased insulin, glycogen, glucose 6-phosphate dehydrogenase, and C-peptide contents, as well as decreased blood sugar, HbA1c, and glucokinase, which are associated with an improvement in diabetic symptoms. Additionally, in histopathological sections, syringic acid was found to reduce pancreatic damage caused by alloxan and stimulate $\beta$-cell regeneration, thus demonstrating its potential to treat diabetes [27, 28].

Yoon et al. [29] demonstrated that $p$-coumaric acid can improve IR and the lipid metabolism of skeletal muscle cells. The administration of $p$-coumaric $(100 \mathrm{mg} / \mathrm{kg} \mathrm{BW})$ in streptozotocin-induced diabetic rats for 30 days can reduce the fasting blood glucose level and $\mathrm{HbAlc}$, as well as increase the insulin level. In addition, $p$-coumaric has been found to have excellent antioxidant ability and can enhance the activity of antioxidant enzymes (catalase, superoxide dismutase, and glutathione peroxidase) of the kidney and liver [23].

Based on the above results, it is speculated that the two phenolic acids--syringic acid and p-coumaric acid-may be the active ingredients for the EAF5-5 fraction to improve glucose uptake in mouse FL83B liver cells and will therefore be developed into drugs or dietary supplements for the treatment of diabetes in the future.

On the other hand, flavonoids isolated from natural substances have been proven to have a variety of physiological effects, such as antioxidation, lowering blood lipids, or antidiabetics [30-32]. RTL contains a variety of flavonoids, such as cirsimaritin, cirsimarin, cirsiliol $4^{\prime}$-glucoside, sorbifolin, or pedalitin $[13,20]$. At present, there are no animal experiments to explore the hypoglycemic effect of cirsimaritin, but cirsimaritin has been found in many natural products with antidiabetic properties $[33,34]$. It is speculated that cirsimaritin may be one of the main active ingredients of flavonoids from the EAF5-5 fraction to improve the glucose uptake capacity of insulin-resistant FL83B hepatocytes.

In this study, three components (syringic acid, $p$ coumaric acid, and cirsimaritin) that were isolated from the EAF5-5 fraction were shown to improve cellular glucose uptake, with $p$-coumaric acid demonstrating the best effect. However, the increase in cellular glucose update is highest when all three compounds were used. We speculated that the hypoglycemic effect of RTL may be the result of multiple active ingredients. One theory has pointed out that if plants can be used to treat diabetes, then their effects can be multiplied by using them in higher concentrations and in combination with the chemical components that are contained in a variety of medicinal plants to cure diabetes 
through distinct mechanisms [35]. Thus, the mechanisms by which RTL improves glucose uptake needs further study.

\section{Conclusions}

Sephadex LH20 gel and MCI gel were used to separate the EA layer of RTL. An evaluation of the glucose uptake in IRinduced FL83B hepatocytes showed that EAF5-5 demonstrated the best activity for improving cell glucose uptake. These activities were eventually linked by HPLC to two phenolic compounds-syringic acid and p-coumaric acid-and the flavonoid compound cirsimaritin. Among the three compounds that significantly improved the activity of cellular glucose uptake, $p$-coumaric acid was the active ingredient with the best effect of increasing glucose uptake although its effect was still not as good as the combined effect of all three compounds and the EAF5-5 fraction. Thus, we speculate that the antidiabetic effect of RTL may be the result of multiple active ingredients, but additional research is needed to confirm this.

\section{Data Availability}

All the data used to support the findings of this study are included within the article.

\section{Conflicts of Interest}

The authors declare that they do not have any conflicts of interest.

\section{Authors' Contributions}

SCS and CYK participated in the design of the study and wrote the protocol and the manuscript. YML participated in the design and discussion of the experiments and the writing of the manuscript. JHX analyzed the results and jointly wrote the manuscript. WCH and JSBW conducted literature searches. WCC, DWH, and YYJ carried out cell experiments as well as TLC and HPLC analysis.

\section{Acknowledgments}

The authors would like to thank the Ministry of Science and Technology of the Republic of China (ROC), Taiwan, for financially supporting this research under contract No. MOST 107-2320-B-003-004-MY3 and academic funding of the Second Affiliated Hospital of Fujian Medical University (serial No. BS201902). Special thanks are due to Professor Y. C. Huang of the Graduate Institute of Pharmacognosy at Taipei Medical University for his guidance in column chromatography analysis.

\section{Supplementary Materials}

Figure S1: thin layer chromatography of eight fractions from RTL-EA by Sephadex LH20 gel column chromatography. Figure S2: concentration effect of RTL-EA fractions on the cell viability of mouse FL83B hepatocytes. Figure S3: thin layer chromatography of five fractions from RTL-EAF5 by MCI gel column chromatography. Figure S4: concentration effect of RTL-EAF5 fractions on the cell viability of mouse FL83B hepatocytes. Figure S5: HPLC chromatogram of phenolic acids from RTL-EAF5-5 fraction. Figure S6: HPLC chromatogram of RTL-EAF5-5 fraction. (Supplementary Materials)

\section{References}

[1] American Diabetes Association (ADA), "Classification and diagnosis of diabetes: standards of medical care in diabetes2020," Diabetes Care, vol. 43, no. 1, pp. S14-S31, 2020.

[2] D. Sola, L. Rossi, G. P. C. Schianca et al., "State of the art paper Sulfonylureas and their use in clinical practice," Archives of Medical Science, vol. 4, no. 4, p. 840, 2015.

[3] F. Alam, M. A. Islam, M. A. Kamal, and S. H. Gan, "Updates on managing type 2 diabetes mellitus with natural products: towards antidiabetic drug development," Current Medicinal Chemistry, vol. 25, no. 39, pp. 5395-5431, 2016.

[4] S. Elavarasi, K. Saravanan, and C. Renuka, "A systematic review on medicinal plants used to treat diabetes mellitus," International Journal of Pharmaceutical, Chemical and Biological Sciences, vol. 3, no. 3, pp. 983-992, 2013.

[5] A. N. M. Mamun-or-Rashid, M. S. Hossain, B. Naim Hassan, M. Kumar Dash, A. Sapon, and M. K. Sen, "A review on medicinal plants with antidiabetic activity," Journal of Pharmacognosy and Phytochemistry, vol. 3, no. 4, pp. 149-159, 2014.

[6] A. N. Singab, F. S. Youssef, and M. L. Ashour, "Medicinal plants with potential antidiabetic activity and their assessment," Medicinal and Aromatic Plant, vol. 3, no. 151, pp. 2167-0412, 2014.

[7] C.-Y. Ko, R.-H. Lin, Y.-M. Zeng et al., "Ameliorative effect of Ruellia tuberosa L. on hyperglycemia in type 2 diabetes mellitus and glucose uptake in mouse C2C12 myoblasts," Food Science \& Nutrition, vol. 6, no. 8, pp. 2414-2422, 2018.

[8] C. Y. Ko, R. H. Lin, Y. M. Lo et al., "Effect of Ruellia tuberosa L. on aorta endothelial damage-associated factors in high-fat diet and streptozotocin-induced type 2 diabetic rats," Food Science \& Nutrition, vol. 7, no. 11, pp. 3742-3750, 2019.

[9] W.-C. Chang, D.-W. Huang, J.-A. Chen, Y.-F. Chang, J. SwiBea Wu, and S.-C. Shen, "Protective effect of Ruellia tuberosa L. extracts against abnormal expression of hepatic detoxification enzymes in diabetic rats," RSC Advances, vol. 8, no. 38, pp. 21596-21605, 2018.

[10] D. W. Huang, Y. M. Lo, W. C. Chang et al., "Alleviative effect of Ruellia tuberosa L. on NAFLD and hepatic lipid accumulation via modulating hepatic de novo lipogenesis in highfat diet plus streptozotocin-induced diabetic rats," Food Science \& Nutrition, vol. 8, no. 10, pp. 1-7, 2020.

[11] C. Phakeovilay, W. Disadee, P. Sahakitpichan et al., "Phenylethanoid and flavone glycosides from Ruellia tuberosa L," Journal of Natural Medicines, vol. 67, no. 1, pp. 228-233, 2013.

[12] V. R. Mohan and K. Vasantha, "GC-MS analysis of bioactive components of tubers of Ruellia tuberosa L. (Acanthaceae)," American Journal of Phytomedicine and Clinical Therapeutics, vol. 2, no. 2, pp. 209-216, 2014.

[13] M. N. Samy, S. Sugimoto, K. Matsunami, H. Otsuka, and M. S. Kamel, "Chemical constituents and biological activities of genus Ruellia," International Journal of Pharmacognosy, vol. 2, no. 6, pp. 270-279, 2015.

[14] J.-J. Chang, M.-J. Hsu, H.-P. Huang, D.-J. Chung, Y.-C. Chang, and C.-J. Wang, "Mulberry anthocyanins inhibit 
oleic acid induced lipid accumulation by reduction of lipogenesis and promotion of hepatic lipid clearance," Journal of Agricultural and Food Chemistry, vol. 61, no. 25, pp. 60696076, 2013.

[15] T. Minh, D. Khang, P. Tuyen et al., "Phenolic compounds and antioxidant activity of phalaenopsis orchid hybrids," Antioxidants, vol. 5, no. 3, p. 31, 2016.

[16] M. F. Beer, F. M. Frank, O. Germán Elso et al., “Trypanocidal and leishmanicidal activities of flavonoids isolated fromStevia satureiifoliavar.satureiifolia," Pharmaceutical Biology, vol. 54, no. 10, pp. 2188-2195, 2016.

[17] D. Shahwara, S. Ullah, M. Ahmad, S. Ullah, N. Ahmad, and M. A. Khan, "Hypoglycemic activity of Ruellia tuberosa linn (Acanthaceae) in normal and alloxan-induced diabetic rabbits," Indian Journal of Pharmaceutical Sciences, vol. 7, pp. 107-115, 2011.

[18] F.-A. Chen, A.-B. Wu, P. Shieh, D.-H. Kuo, and C.-Y. Hsieh, "Evaluation of the antioxidant activity of Ruellia tuberosa," Food Chemistry, vol. 94, no. 1, pp. 14-18, 2006.

[19] D. R. Wulan, E. P. Utomo, and C. Mahdi, "Antidiabetic activity of Ruellia tuberosa L., role of -amylase inhibitor: in silico, in vitro, and in vivo approaches," Biochemistry Research International, vol. 2015, Article ID 349261, 9 pages, 2015.

[20] C. F. Lin, Y. L. Huang, L. Y. Cheng, S. J. Sheu, and C. C. Chen, "Bioactive flavonoids from Ruellia tuberosa," The Journal of Chinese Medicine, vol. 17, no. 3, pp. 103-109, 2006.

[21] O. Cikman, O. Soylemez, O. F. Ozkan et al., "Antioxidant activity of syringic acid prevents oxidative stress in l-arginineInduced acute pancreatitis: an experimental study on rats," International Surgery, vol. 100, no. 5, pp. 891-896, 2015.

[22] S. H. Sharma, D. R. Chellappan, P. Chinnaswamy, and S. Nagarajan, "Protective effect of $p$-coumaric acid against 1,2 dimethylhydrazine induced colonic preneoplastic lesions in experimental rats," Biomedicine \& Pharmacotherapy, vol. 94, pp. 577-588, 2017.

[23] M. DellaGreca, L. Previtera, R. Purcaro, and A. Zarrelli, "Cinnamic ester derivatives from Oxalis pes-caprae (Bermuda buttercup)\#," Journal of Natural Products, vol. 70, no. 10, pp. 1664-1667, 2007.

[24] M. DellaGreca, A. Fiorentino, P. Monaco, L. Previtera, and A. Zarrelli, "A new dimeric 9,10-dihydrophenanthrenoid from the rhizome of Juncus acutus," Tetrahedron Letters, vol. 43, no. 14, pp. 2573-2575, 2002.

[25] M. DellaGreca, A. Fiorentino, M. Isidori, L. Previtera, F. Temussi, and A. Zarrelli, "Benzocoumarins from the rhizomes of Juncus acutus," Tetrahedron, vol. 59, no. 26, pp. 4821-4825, 2003.

[26] R. Vinayagam, M. Jayachandran, and B. Xu, "Antidiabetic effects of simple phenolic acids: a comprehensive review," Phytotherapy Research, vol. 30, no. 2, pp. 184-199, 2016.

[27] J. Muthukumaran, S. Srinivasan, R. S. Venkatesan, V. Ramachandran, and U. Muruganathan, "Syringic acid, a novel natural phenolic acid, normalizes hyperglycemia with special reference to glycoprotein components in experimental diabetic rats," Journal of Acute Disease, vol. 2, no. 4, pp. 304-309, 2013.

[28] S. Srinivasan, J. Muthukumaran, U. Muruganathan, R. S. Venkatesan, and A. M. Jalaludeen, "Antihyperglycemic effect of syringic acid on attenuating the key enzymes of carbohydrate metabolism in experimental diabetic rats," Biomedicine \& Preventive Nutrition, vol. 4, no. 4, pp. 595-602, 2014.

[29] S.-A. Yoon, S.-I. Kang, H.-S. Shin et al., "p-Coumaric acid modulates glucose and lipid metabolism via AMP-activated protein kinase in L6 skeletal muscle cells," Biochemical and Biophysical Research Communications, vol. 432, no. 4, pp. 553-557, 2013.

[30] V. Amalan and N. Vijayakumar, "Antihyperglycemic effect of p-coumaric acid on streptozotocin induced diabetic rats," Indian Journal of Applied Research, vol. 5, pp. 10-13, 2015.

[31] X. Wang, Q. Liu, H. Zhu et al., "Flavanols from the Camellia sinensis var. assamica and their hypoglycemic and hypolipidemic activities," Acta Pharmaceutica Sinica B, vol. 7, no. 3, pp. 342-346, 2017.

[32] M. Abbas, F. Saeed, F. M. Anjum et al., "Natural polyphenols: an overview," International Journal of Food Properties, vol. 20, no. 8, pp. 1689-1699, 2017.

[33] E. G. E. Helal, N. Abou-Aouf, and A. S. M. Khattab, “A possible hypoglycemic and antioxidant effect of herbal mixture extraction in diabetic rats," The Egyptian Journal of Hospital Medicine, vol. 58, pp. 109-119, 2015.

[34] H. Y. Al Ati, G. A. Fawzy, A. A. El Gamal et al., "Phytochemical and biological evaluation of Buddleja polystachya growing in Saudi Arabia," Pakistan Journal of Pharmaceutical Sciences, vol. 28, no. 4, pp. 1533-1540, 2015.

[35] U. F. Ezuruike and J. M. Prieto, "The use of plants in the traditional management of diabetes in Nigeria: pharmacological and toxicological considerations," Journal of Ethnopharmacology, vol. 155, no. 2, pp. 857-924, 2014. 